\title{
Plasmacytoid Dendritic Cells: The Potential of Synergistic Crosstalk between Innate and Adaptive Immunity
}

\begin{abstract}
Tania Rahman $1,2 *$
${ }^{1}$ Department of Biochemistry and Molecular Biology, Bio21 Molecular Science and Biotechnology Institute, University of Dhaka, Bangladesh

${ }^{2}$ Department of Microbiology and Immunology, University of Melbourne at the Peter Doherty Institute for Infection and Immunity, Australia
\end{abstract}

*Corresponding author: Tania Rahman, Associate Professor, Department of Biochemistry and Molecular Biology, University of Dhaka, Bangladesh, Tel: +88-01917285696; Email: tania.rahman@du.ac.bd

\section{Abstract}

In rodents, a combination of innate and adaptive immune responses is critical in controlling infection. The adaptive immune components, comprising B cells, CD4 T cells, CD8 T cells, pathogen specific secretory antibodies, and Treg cells have been exhibited to play an indispensable role in comprising and eliminating various infections. Also, there are several innate immune components that help to control infections, such as soluble mediators, lymphotoxin (LT) signaling in innate epithelial cells, TLRs, signaling adaptor MyD88, inflammasomes, CXCL9, P-selectin glycoprotein ligand-1 (PSGL-1). Little is known about the molecules that mediate the crosstalk between innate and adaptive immune response during infection. The work presented here has unravelled the potential of plasmacytoid dendritic cells (pDC) in the immune system in providing the first line of defense during natural infection as well as subsequent adaptive host defense against pathogens. In this work the potential that pDC might have in infection has been investigated in order to understand the basis of the development of protective and pathological responses during infection. These findings would help set up future avenues of research to elucidate a key mechanism of action of these cells and provide new therapeutic insights.

Keywords: Type I IFN-producing Cells, CD4 T Cells, CD8 T Cell, Treg Cell, Th17 Response, NK Cell, Soluble Mediators, Secretory Antibodies

Abbreviations: LT: Lymphotoxin; PSGL-1: P-Selectin Glycoprotein Ligand-1; pDC: plasmacytoid Dendritic Cells; HIV: Human Immunodeficiency Virus; HSV: Herpes
Simplex Virus; MCMV: Murine Cytomegalo Virus; LCMV: Lymphocytic Choriomeningitis Virus. 


\section{Journal of Infectious Diseases \& Travel Medicine}

\section{Introduction}

pDC are a unique population of bone marrow derived leukocytes that are developmentally and functionally distinct from conventional DCs $[1,2]$. In the steady state, pDC reside primarily in the lymphoid organs and are recruited by infection and inflammatory mediators [2-4]. For instance, accumulations of pDC are prevalent in the skin in psoriasis [5] and SLE [6], in the mucosal tissues in allergic rhinitis [7], in the lungs in pneumonia [8] and respiratory syncytial virus [9], in the mucosal space in colon and mesenteric LN in IBDs [10], in vagina in genital HSV-2 infection [11]. pDC can differentiate into antigenpresenting dendritic cells as a result of their link to several cellular receptors that promptly detect nucleic acids in pathogens $[2,12]$. These powerful networks of molecular and cellular events permit pDC to bridge the innate and adaptive immune systems resulting in a concerted pathogen response.

\section{pDC during Infection}

As the major type I IFN-producing cells in the innate immune system, $\mathrm{pDC}$ respond to a wide range of viruses, including human immunodeficiency virus type I (HIV-1), influenza virus, sendai virus, herpes simplex virus (HSV), murine cytomegalovirus (MCMV) and lymphocytic choriomeningitis virus (LCMV) etc [13]. However, their role in non-viral diseases was not much appreciated until recently a handful of studies have begun to explore the contribution of $\mathrm{pDC}$ to bacterial immune responses and autoimmunity. For instance, pDC produce IFN-I upon recognition of bacterial RNA $[14,15]$ and when exposed to Borrelia burgdorferi in vitro [16], they can activate CD4 T cells via antigen presentation and IL12 secretion in response to toxoplasma infection [17]. Also, pDC can suppress induction of CD4 T cells and initiate CD8 $\mathrm{T}$ cell responses [18]. pDC can infiltrate the mesenteric LN and spleen of Listeria-infected mice [19], although they are not responsible for the elevated systemic IFN-I levels [20] that interfere with the clearance of Listeria [21]. pDC have also been shown to drive Th17 responses in patients with GVHD [22] and pDC produce LN homing of NK cells [23]. The potential of $\mathrm{pDC}$ to induce Treg has been illustrated in numerous disease models [24-27]. Depletion of pDC results in an upregulation of $\mathrm{T}$ cell immune responses, increased production of thymic regulatory $\mathrm{T}$ (Treg) cells, fewer Treg cells in the gut, decreased generation of induced Treg cells and an increased frequency Th17 cells [28]. Recently, pDC have been found to be associated with the development of central tolerance in the thymus [29]. This work supports earlier studies that suggest a tolerogenic role for $\mathrm{pDC}$ in some circumstances.

\section{pDC a Specialized Type I Interferon Secreting Cells}

pDC, a distinctive subset of DCs is able to secrete type I IFNs more quickly than other cell types to a large array of viral and non-viral molecules [30-32]. Though comprising only $0.2-0.8 \%$ of human blood cells, pDC are able to produce more than $95 \%$ of type I IFNs by peripheral blood mononuclear cells following viral insult [33,34]. pDC are proficient in secreting up to $10 \mathrm{pg} /$ cell of type I IFN, producing them 10-100-fold more than other cell types including moDC [35]. Within $6 \mathrm{~h}$ of activation, human pDC contribute $60 \%$ of their transcriptional activity to secrete type I IFNs, comprising entire transcripts of nineteen diverse subclasses of type I IFN [36]. pDC express a wide range of ifna genes compared to other professional antigen-presenting cells (APCs). Human pDC express the entire subtypes of type I IFNs including IFN- $\alpha$, IFN- $\beta$, IFN- $\gamma$, IFN- $\omega$, and IFN- $\tau$ [1]. Ablation of pDC in vivo abolished the IFN- $\alpha$ secretion in response to cytomegalovirus infection in mice $[37,38]$.

\section{Role of pDC as a Linker of Innate and Adaptive Immunity}

Type I IFNs produced by pDC induce a cellular antiviral response and recruits a network of molecular and cellular events and thus linking the innate with adaptive immunity [39] (Figure 1). Type I IFNs activate NK cell cytolytic activity, however, protect uninfected cells from NK cell-mediated lysis $[40,41]$. pDC induce production of IFN $\gamma$ in NK cells via IL-12 secretion [42]. Type I interferons released by $\mathrm{pDC}$ cause $\mathrm{T}$ cells to produce IL-10, IFN $\gamma$ and also induce a Th1 polarization and thus promote clearance of intracellular pathogens $[43,44]$. Type I interferons can upregulate early activation markers CD69 on T cells $[45,46]$ and generation of longterm antitumor immune response [47]. Through the production of both type I interferons and IL-6, pDC promote human B cells to differentiate into antibody secreting plasma cells facilitating the production of antiviral antibodies $[48,49]$. Plasma cells induced by pDC predominantly secrete IgG rather than IgM, suggesting that pDC may trigger memory B cells $[50,51]$. Type I IFNs can increase cross-presentation during viral infection, and induce antigen-specific CD8+ $\mathrm{T}$ cells, and the differentiation of naive $\mathrm{T}$ cells into Th1 cells using the STAT1, STAT3 activation and T-bet expression [52-54]. Type I IFNs trigger immature cDCs to produce IL-12, IL15 , IL-18, and IL-23, cytokines that are not secreted by human pDC $[55,56]$. pDC are likewise competent producers of chemokines, mostly CCL3, which can aid 


\section{Journal of Infectious Diseases \& Travel Medicine}

them to trigger additional leukocytes to inflamed tissues $\quad[57,58]$.

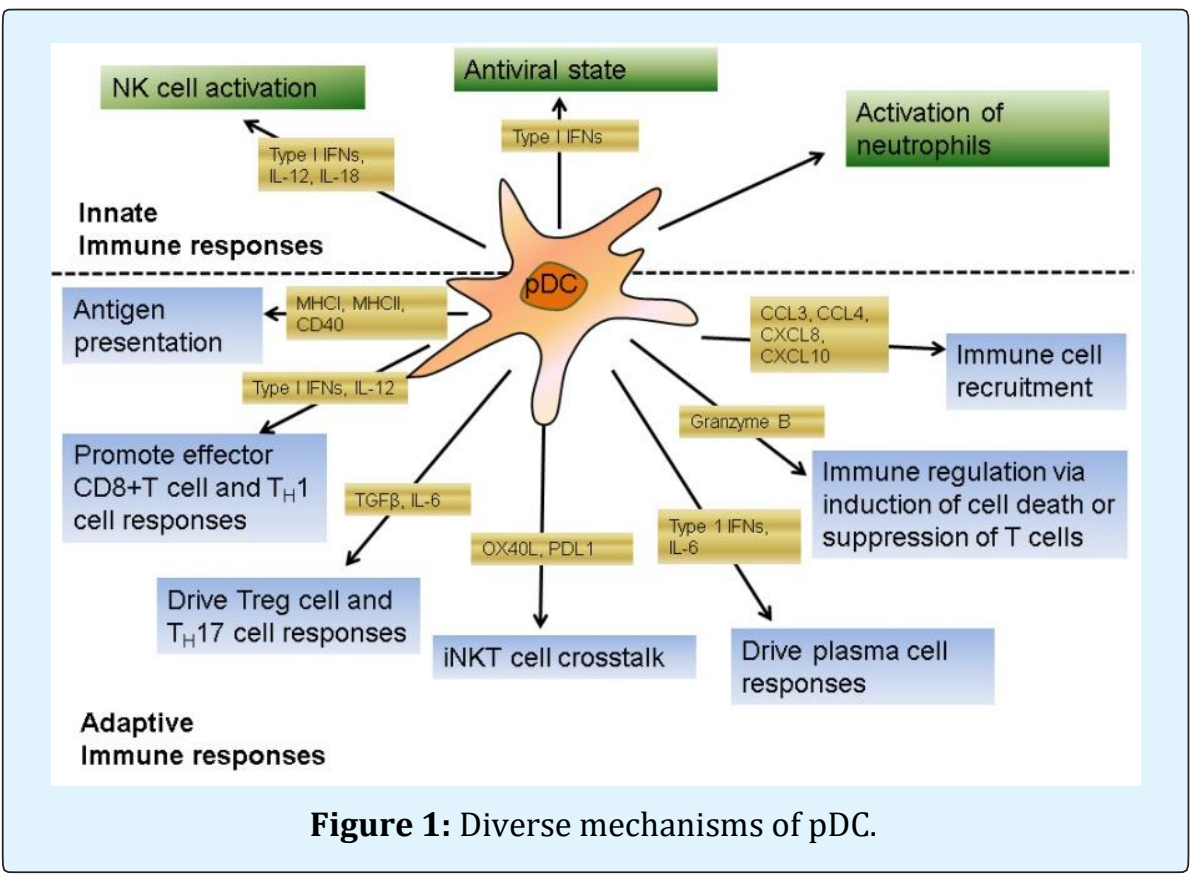

pDC are important drivers for both innate and adaptive immune responses. pDC production of MHC class I, Class II and costimulatory molecule CD40 enable pDCs to present antigen to CD4 cells. pDC production of type I IFNs and IL-12 enable pDCs to promote effector CD8 T cells as well as polarization of $\mathrm{CD} 4$ cells to Th1 responses.pDC production of TGF $\beta$ and IL- 6 drive either Treg or Th17 cell responses. The crosstalk between pDCs and NKT cells occur via OX40L and PDL1 ligand. pDC production of type 1 IFNs and IL- 6 drive plasma cell responses. Granzyme B enablepDCs to induce apoptosis or cell death as well as suppression of T cells. pDCs induce chemokines including CCL3, CCL4, CCL8 and CXCL10 which can attract other immune cells to the site of infection.pDC can activate neutrophils. pDC production of type 1 IFNs can produce an antiviral adaptive immune responses.pDC production of type 1 IFNs, IL-12 and IL-18 can activate NK cells.

\section{Concluding Remarks}

The role of pDC has been exhibited in two distinct immune systems, one of which gives rise to the other. $\mathrm{pDC}$, which are typically believed to be as antiviral, enact a central role in host control of bacterial infections. The findings in this study can be extended to other undiagnosed bacterial and parasite infections. Also, additional studies are required on pDC in different type of infections to hypothesize extended knowledge on the vivid character of these cells and mechanisms contributing to immunity against various pathogens.

\section{References}

1. Lande R, Gilliet M (2010) Plasmacytoid dendritic cells: key players in the initiation and regulation of immune responses. Ann N Y Acad Sci 1183: 89-103.

2. Villadangos JA, Young L (2008) Antigen-presentation properties of plasmacytoid dendritic cells. Immunity 29(3): 352-361.

3. Asselin-Paturel C, Boonstra A, Dalod M, Durand I, Yessaad N, et al. (2001) Mouse type I IFN-producing cells are immature APCs with plasmacytoid morphology. Nat Immunol 2(12): 1144-1150.

4. Sozzani S, Vermi W, Del Prete A, Facchetti F (2010) Trafficking properties of plasmacytoid dendritic cells in health and disease. Trends Immunol 31(7): 270277.

5. Glitzner E, Korosec A, Brunner PM, Drobits B, Amberg $\mathrm{N}$, et al. (2014) Specific roles for dendritic cell subsets during initiation and progression of psoriasis. EMBO Mol Med 6(10): 1312-1327. 


\section{Journal of Infectious Diseases \& Travel Medicine}

6. Ronnblom L (2011) The type I interferon system in the etiopathogenesis of autoimmune diseases. Ups J Med Sci 116(4): 227-237.

7. Jahnsen FL, Lund-Johansen F, Dunne JF, Farkas L, Haye R, et al. (2000) Experimentally induced recruitment of plasmacytoid (CD123high) dendritic cells in human nasal allergy. J Immunol 165(7): 40624068 .

8. Ang DK, Oates CV, Schuelein R, Kelly M, Sansom FM, et al. (2010) Cutting edge: pulmonary Legionella pneumophila is controlled by plasmacytoid dendritic cells but not type I IFN. J Immunol 184(10): 54295433.

9. Wang H, Peters N, Schwarze J (2006) Plasmacytoid dendritic cells limit viral replication, pulmonary inflammation, and airway hyper responsiveness in respiratory syncytial virus infection. J Immunol 177(9): 6263-6270.

10. Baumgart DC, Metzke D, Guckelberger O, Pascher A, Grötzinger C, et al. (2011) Aberrant plasmacytoid dendritic cell distribution and function in patients with Crohn's disease and ulcerative colitis. Clin Exp Immunol 166(1): 46-54.

11. Lund JM, Linehan MM, Iijima N, Iwasaki A (2006) Cutting Edge: Plasmacytoid dendritic cells provide innate immune protection against mucosal viral infection in situ. J Immunol 177(11): 7510-7514.

12. Lombardi VC, Khaiboullina SF (2014) Plasmacytoid dendritic cells of the gut: relevance to immunity and pathology. Clin Immunol 153(1): 165-177.

13. Tang F, Du QM, Liu YJ (2010) Plasmacytoid dendritic cells in antiviral immunity and autoimmunity. Sci China-Life Sci 53(2): 172-182.

14. Chiu YH, Macmillan JB, Chen ZJ (2009) RNA polymerase III detects cytosolic DNA and induces type I interferons through the RIG-I pathway. Cell 138(3): 576-591.

15. Eberle F, Sirin M, Binder M, Dalpke AH (2009) Bacterial RNA is recognized by different sets of immunoreceptors. Eur J Immunol 39(9): 2537-2547.

16. Petzke MM, Brooks A, Krupna MA, Mordue D, Schwartz I (2009) Recognition of Borrelia burgdorferi, the Lyme disease spirochete, by TLR7 and TLR9 induces a type I IFN response by human immune cells. J Immunol 183(8): 5279-5292.
17. Pepper M, Dzierszinski F, Wilson E, Tait E, Fang Q, et al. (2008) Plasmacytoid dendritic cells are activated by Toxoplasma gondii to present antigen and produce cytokines. J Immunology 180(9): 6229-6236.

18. Takagi H, Fukaya T, Eizumi K, Sato Y, Sato K, et al. (2011) Plasmacytoid Dendritic Cells Are Crucial for the Initiation of Inflammation and T Cell Immunity in Vivo. Immunity 35(6): 958-971.

19. Tam MA, Wick MJ (2006) Differential expansion, activation and effector functions of conventional and plasmacytoid dendritic cells in mouse tissues transiently infected with Listeria monocytogenes. Cell Microbiol 8(7): 1172-1187.

20. Stockinger S, Kastner R, Kernbauer E, Pilz A, Westermayer S, et al. (2009) Characterization of the interferon-producing cell in mice infected with Listeria monocytogenes. PLoS Pathog 5(3): 1000355.

21. Rayamajhi M, Humann J, Penheiter K, Andreasen K, Lenz LL (2010) Induction of IFN-alphabeta enables Listeria monocytogenes to suppress macrophage activation by IFN-gamma. J Exp Med 207(2): 327-337.

22. Bossard C, Malard F, Arbez J, Chevallier P, Guillaume T, et al. (2012) Plasmacytoid dendritic cells and Th17 immune response contribution in gastrointestinal acute graft-versus-host disease. Leukemia 26(7): 1471-1474.

23. Romagnani C, Della Chiesa M, Kohler S, Moewes B, Radbruch A, et al. (2005) Activation of human NK cells by plasmacytoid dendritic cells and its modulation by CD4+ T helper cells and CD4+ CD25hi T regulatory cells. Eur J Immunol 35(8): 2452-2458.

24. De Heer HJ, Hammad H, Soullié T, Hijdra D, Vos N, et al. (2004) Essential role of lung plasmacytoid dendritic cells in preventing asthmatic reactions to harmless inhaled antigen. J Exp Med 200(1): 89-98.

25. Ochando JC, Homma C, Yang Y, Hidalgo A, Garin A, et al. (2006) Alloantigen-presenting plasmacytoid dendritic cells mediate tolerance to vascularized grafts. Nat Immunol 7(6): 652-662.

26. Goubier A, Dubois B, Gheit H, Joubert G, Villard-Truc F, et al. (2008) Plasmacytoid dendritic cells mediate oral tolerance. Immunity 29(3): 464-475.

27. Adalid-Peralta L, Fragoso G, Fleury A, Sciutto E (2011) Mechanisms underlying the induction of regulatory $\mathrm{T}$ cells and its relevance in the adaptive immune 


\section{Journal of Infectious Diseases \& Travel Medicine}

response in parasitic infections. Int J Biol Sci 7(9): 1412-1426.

28. Takagi H, Fukaya T, Eizumi K, Sato Y, Sato K, et al. (2011) Plasmacytoid dendritic cells are crucial for the initiation of inflammation and $\mathrm{T}$ cell immunity in vivo. Immunity 35(6): 958-971.

29. Hadeiba H, Lahl K, Edalati A, Oderup C, Habtezion A, et al. (2012) Plasmacytoid dendritic cells transport peripheral antigens to the thymus to promote central tolerance. Immunity 36(3): 438-450.

30. Schettini J, Mukherjee P (2008) Physiological role of plasmacytoid dendritic cells and their potential use in cancer immunity. Clin Dev Immunol 2008: 106321.

31. Liu YJ (2005) IPC: professional type 1 interferonproducing cells and plasmacytoid dendritic cell precursors. Annu Rev Immunol 23: 275-306.

32. Mathan TS, Figdor CG, Buschow SI (2013) Human plasmacytoid dendritic cells: from molecules to intercellular communication network. Front Immunol 4: 372 .

33. Fitzgerald-Bocarsly $P$, Feldman $M$, Mendelsohn $M$, Curl S, Lopez C (1988) Human mononuclear cells which produce interferon-alpha during NK(HSV-FS) assays are HLA-DR positive cells distinct from cytolytic natural killer effectors. J Leukoc Biol 43(4): 323-334.

34. Fitzgerald-Bocarsly P, Dai J, Singh S (2008) Plasmacytoid dendritic cells and type I IFN: 50 years of convergent history. Cytokine Growth Factor Rev 19(1): 3-19.

35. Ito T, Kanzler H, Duramad O, Cao W, Liu YJ (2006) Specialization, kinetics, and repertoire of type 1 interferon responses by human plasmacytoidpredendritic cells. Blood 107(6): 24232431.

36. Siegal FP, Kadowaki N, Shodell M, Fitzgerald-Bocarsly PA, Shah K, et al. (1999) The nature of the principal type 1 interferon-producing cells in human blood. Science 284(5421): 1835-1837.

37. Barchet W, Cella M, Odermatt B, Asselin-Paturel C, Colonna M, et al. (2002) Virus-induced interferon alpha production by a dendritic cell subset in the absence of feedback signaling in vivo. J Exp Med 195(4): 507-516.
38. Reizis B, Bunin A, Ghosh HS, Lewis KL, Sisirak V, et al. (2011) Plasmacytoid Dendritic Cells: Recent Progress and Open Questions. Annu Rev Immunol 29: 163-183.

39. Kadowaki N, Antonenko S, Lau JY, Liu YJ (2000) Natural interferon alpha/beta-producing cells link innate and adaptive immunity. J Exp Med 192(2): 219-226.

40. Hanabuchi S, Watanabe N, Wang YH, Wang YH, Ito T, et al. (2006) Human plasmacytoid predendritic cells activate NK cells through glucocorticoid-induced tumor necrosis factor receptor-ligand (GITRL). Blood 107(9): 3617-3623.

41. Bandyopadhyay S, Perussia B, Trinchieri G, Miller DS, Starr SE (1986) Requirement for HLA-DR+ accessory cells in natural killing of cytomegalovirus-infected fibroblasts. J Exp Med 164(1): 180-195.

42. Colonna M, Trinchieri G, Liu YJ (2004) Plasmacytoid dendritic cells in immunity. Nat Immunol 5(12): 1219-1226.

43. Cella M, Facchetti F, Lanzavecchia A, Colonna M (2000) Plasmacytoid dendritic cells activated by influenza virus and CD40L drive a potent TH1 polarization. Nat Immunol 1(4): 305-310.

44. Visperas A, Do J, Min B (2014) Cellular factors targeting APCs to modulate adaptive T cell immunity. J Immunol Res 2014: 750374.

45. Agnello D, Lankford CS, Bream J, Morinobu A, Gadina M, et al. (2003) Cytokines and transcription factors that regulate $\mathrm{T}$ helper cell differentiation: new players and new insights. J Clin Immunol 23(3): 147161.

46. Hemmi M, Tachibana M, Tsuzuki S, Shoji M, Sakurai F, et al. (2014) The early activation of CD8+ T cells is dependent on type I IFN signaling following intramuscular vaccination of adenovirus vector. Biomed Res Int 2014: 158128.

47. Belardelli F, Gresser I (1996) The neglected role of type I interferon in the T-cell response: implications for its clinical use. Immunol Today 17(8): 369-372.

48. Jego G, Palucka AK, Blanck JP, Chalouni C, Pascual V, et al. (2003) Plasmacytoid dendritic cells induce plasma cell differentiation through type I interferon and interleukin 6. Immunity 19(2): 225-234. 
49. Gujer C, Sandgren KJ, Douagi I, Adams WC, Sundling C, et al. (2011) IFN-alpha produced by human plasmacytoid dendritic cells enhances $\mathrm{T}$ celldependent naive $\mathrm{B}$ cell differentiation. J Leukoc Biol 89(6): 811-821.

50. Poeck H, Wagner M, Battiany J, Rothenfusser S, Wellisch D, et al. (2004) Plasmacytoid dendritic cells, antigen, and $\mathrm{CpG}-\mathrm{C}$ license human B cells for plasma cell differentiation and immunoglobulin production in the absence of T-cell help. Blood 103(8): 30583064 .

51. Crampton SP, Voynova E, Bolland S (2010) Innate pathways to B-cell activation and tolerance. Ann N Y Acad Sci 1183: 58-68.

52. Kolumam GA, Thomas S, Thompson LJ, Sprent J, Murali-Krishna K (2005) Type I interferons act directly on CD8 T cells to allow clonal expansion and memory formation in response to viral infection. J Exp Med 202(5): 637-650.

53. Le Bon A, Etchart N, Rossmann C, Ashton M, Hou S, et al. (2003) Cross-priming of CD8+ T cells stimulated by virus-induced type I interferon. Nat Immunol 4(10): 1009-1015.
54. Tang F, Du Q, LiuYJ (2010) Plasmacytoid dendritic cells in antiviral immunity and autoimmunity. Sci China Life Sci 53(2): 172-182.

55. Santini SM, Lapenta C, Logozzi M, Parlato S, Spada M, et al. (2000) Type I interferon as a powerful adjuvant for monocyte-derived dendritic cell development and activity in vitro and in Hu-PBL-SCID mice. J Exp Med 191(10): 1777-1788.

56. Paquette RL, Hsu NC, Kiertscher SM, Park AN, Tran L, et al. (1998) Interferon-alpha and granulocytemacrophage colony-stimulating factor differentiate peripheral blood monocytes into potent antigenpresenting cells. J Leukoc Biol 64(3): 358-367.

57. Krug A, Uppaluri R, Facchetti F, Dorner BG, Sheehan $\mathrm{KC}$, et al. (2002) IFN-producing cells respond to CXCR3 ligands in the presence of CXCL12 and secrete inflammatory chemokines upon activation. J Immunol 169(11): 6079-6083.

58. Megjugorac NJ, Young HA, Amrute SB, Olshalsky SL, Fitzgerald-Bocarsly P (2004) Virally stimulated plasmacytoid dendritic cells produce chemokines and induce migration of $\mathrm{T}$ and NK cells. J Leukoc Biol 75(3): 504-514. 